\title{
On $f(R)$ Theories in Two-Dimensional Spacetime
}

\author{
M. A. Ahmed \\ Physics Department, Kuwait University, P.O. Box 5969, Safat 13060, Kuwait \\ Correspondence should be addressed to M. A. Ahmed, mkarim.ahmed@gmail.com
}

Received 22 June 2011; Revised 26 October 2011; Accepted 3 December 2011

Academic Editor: Ashok Chatterjee

Copyright $\odot 2012$ M. A. Ahmed. This is an open access article distributed under the Creative Commons Attribution License, which permits unrestricted use, distribution, and reproduction in any medium, provided the original work is properly cited.

In recent years, theories in which the Einstein-Hilbert Lagrangian is replaced by a function $f(R)$ of the Ricci Scalar have been extensively studied in four-dimensional spacetime. In this paper we carry out an analysis of such theories in two-dimensional spacetime with focus on cosmological implications. Solutions to the cosmological field equations are obtained and their properties are analysed. Inflationary solutions are also obtained and discussed. Quantization is then carried out, the Wheeler-DeWitt equation is set up, and its exact solutions are obtained.

\section{Introduction}

Attempts to modify the theory of general relativity, by including higher-order invariants in the action, started not too long after its inception $[1,2]$. Later the nonrenormalizability of general relativity gave impetus to the inclusion of higher-order terms in the action $[3,4]$. More recently it was shown that when quantum corrections are taken into consideration, higher order curvature invariants need to be added to the low-energy gravitational action $[5,6]$. Such considerations further increased the interest in constructing theories in which the Einstein-Hilbert action is extended by the inclusion of higher-order curvature invariants with respect to the Ricci Scalar. Our interest here is in the so-called $f(R)$ theories of gravity. In these theories the Lagrangian in the Einstein-Hilbert action

$$
I_{G}^{\prime}=-\frac{1}{2 \kappa} \int d^{4} x \sqrt{-g} R,
$$

where $\kappa=8 \pi G, G$ is the gravitational constant, $g$ is the determinant of the metric tensor and $R$ is the Ricci scalar (in units $c=\hbar=1$ ), is generalized to become

$$
I^{\prime}=-\frac{1}{2 \kappa} \int d^{4} x \sqrt{-g} f(R) .
$$

In (2) $f(R)$ is a general function of $R$ [7]. Our focus here is on the cosmological aspects of $f(R)$ theories.

Now in another direction, the quest for quantum theory of gravity has led to the study of the simpler case of gravitational theory in two-dimensional spacetime. Such a spacetime provides an interesting arena in which to explore some fundamental aspects of both classical and quantum gravity. The reduction in the degrees of freedom greatly simplifies the analysis of the field equations. This leads to appreciable understanding of several problems in gravity theory. In two-dimensional spacetime, the two-dimensional gravitational constant $G_{2}$ is dimensionless and formally the theory with the bare action

$$
I_{G}=-\frac{1}{2 g_{N}} \int d^{2} x \sqrt{-g} R,
$$

where $g_{N}=8 \pi G_{2}$, is power counting renormalizable in perturbation theory. However the Einstein-Hilbert action term is purely topological in two dimensions. In fact in two spactime dimensions, the curvature tensor $R_{\mu \nu \lambda \rho}$ has only one independent component since all nonzero components may be obtained by symmetry from $R_{0101}$. Equivalently the curvature tensor may be written in terms of the curvature scalar [8]:

$$
R_{\mu \nu \lambda \rho}=\frac{1}{2} R\left(g_{\mu \lambda} g_{\nu \rho}-g_{\mu \rho} g_{\nu \lambda}\right)
$$

so that $R$ alone completely characterizes the local geometry. Equation (4) implies that

$$
R_{\mu \nu}=\frac{1}{2} g_{\mu \nu} R
$$


so that the Einstein tensor $G_{\mu \nu}=R_{\mu \nu}-(1 / 2) g_{\mu \nu} R$, vanishes identically and the usual Einstein equations are meaningless in two dimensions. This led to various models for gravity in two-dimensional spacetime being proposed [9]. Of special interest are those models that involve a scalar field, the dilaton, in the action [9-12]. We have previously studied some aspects of classical and quantum cosmology in twodimensional dilaton gravity models $[13,14]$. In the present work we study $f(R)$ theories as an alternative way to formulate gravitational theory in two-dimensional spactime and explore some of their cosmological implications.

In Section 2 we set up the $f(R)$ gravity theory in two-dimensional spacetime and derive the general field equations. We then specialize to the case of the FriedmannRobertson-Walker metric and obtain the field equations with matter treated as a perfect fluid. Section 3 is devoted to obtaining solutions to the cosmological field equations under various conditions of matter or radiation dominance. Properties of these solutions are discussed in Section 4. In particular, conditions for ensuring cosmic acceleration and solving the horizon problem are elucidated. Inflation is discussed in Section 5 and solutions to the field equations in the absence of matter or radiation are obtained and their properties are discussed. In Section 6 we carry out the quantization. We establish the Wheeler-DeWitt equation and obtain its solutions. In Section 7 we offer some concluding remarks.

\section{Field Equations}

We write the two-dimensional action for $f(R)$ gravity as

$$
I=I_{G}+I_{M}
$$

where

$$
I_{G}=-\frac{1}{2 g_{N}} \int d^{2} x \sqrt{-g} f(R)
$$

is the gravitational action and $I_{M}$ is the matter action [15]. The field equations can be derived by varying the action with respect to the metric tensor $g_{\mu_{\nu}}$. Upon noting that the stressenergy tensor is defined by

$$
\delta I_{M}=\frac{1}{2} \int d^{2} x \sqrt{-g} T^{\mu \nu} \delta g_{\mu \nu}
$$

we derive the following field equation:

$f^{\prime}(R) R_{\mu \nu}-\frac{1}{2} g_{\mu \nu} f(R)-g_{\mu \nu} \square f^{\prime}(R)+\nabla_{\mu} \nabla_{\mu} f^{\prime}(R)=-g_{N} T_{\mu_{\nu}}$.

In (4) $R_{\mu \nu}$ is the Ricci tensor, the prime denotes the differentiation with respect to $R$, and the operator $\square$ is defined by

$$
\square f^{\prime}(R)=\frac{1}{\sqrt{-g}} \partial_{\mu}\left(\sqrt{-g} g^{\mu \nu} \partial_{\nu} f^{\prime}(R)\right) .
$$

Using (5) we can write (9) as

$$
\frac{1}{2} g_{\mu \nu}\left(f^{\prime}(R) R-f(R)\right)-g_{\mu \nu} \square f^{\prime}(R)+\nabla_{\mu} \nabla_{\nu} f^{\prime}(R)=-g_{N} T_{\mu_{\nu}} .
$$

In the following we will concern with cosmological implications of (11). For this purpose will adopt the FriedmanRobertson-Walker (FRW) metric which in two-dimensional spacetime reads $(c=1)$

$$
d s^{2}=-d t^{2}+\frac{a^{2}(t)}{1-k x^{2}} d x^{2},
$$

in terms of the comoving coordinates $x$ and $t$. The quantity $a(t)$ is the usual time-dependent cosmic scale factor. A change of variable $d x^{2} /\left(1-k x^{2}\right) \rightarrow d x^{2}$ leads to

$$
d s^{2}=-d t^{2}+a^{2}(t) d x^{2}
$$

Thus in two dimensions the time evolution of $a(t)$ is not affected by the value of $k=0, \pm 1$ corresponding to the three different cosmological models [16]. This is unlike the four-dimensional case. The values $k=0,-1$ still describe spatially open flat and hyperbolic universe respectively, while $k=1$ describes a closed universe. The stress-energy tensor of the homogeneous isotropic universe is taken to be that of a perfect fluid:

$$
T_{\mu \nu}=p g_{\mu \nu}+(p+\rho) U_{\mu} U_{\nu}
$$

where $p$ is the pressure, $\rho$ is the energy density, and $U^{\mu}$ is the comoving velocity. Using (13) and (14) we obtain from (11) the following two independent cosmological field equations:

$$
\begin{aligned}
& \frac{1}{2}\left(R f^{\prime}(R)-f(R)\right)+\frac{\dot{a}}{a} \partial_{t} f^{\prime}(R)=g_{N} \rho, \\
& \frac{1}{2}\left(R f^{\prime}(R)-f(R)\right)+\partial_{t}^{2} f^{\prime}(R)=-g_{N} p,
\end{aligned}
$$

where we use the dot as well as $\partial_{t}$ to indicate differentiation with respect to time. We note that if $f(R)$ is expressed as a sum of powers $R^{n}$ of $R$, then a term linear in $R$ would cancel out in the bracketed terms in (15) and would not contribute to the derivative terms either. Hence it has no effect on the dynamics. The stress-energy tensor obeys the conservation law:

$$
\nabla^{\alpha} T_{\alpha_{\beta}}=0
$$

and this, for a perfect fluid, gives rise to the following two equations:

$$
\begin{gathered}
U^{\alpha} \nabla_{\alpha} \rho+(p+\rho) \nabla^{\alpha} U_{\alpha}=0, \\
(p+\rho) U^{\alpha} \nabla_{\alpha} U_{\beta}+\left(g_{\alpha \beta}+U_{\alpha} U_{\beta}\right) \nabla^{\alpha} p=0 .
\end{gathered}
$$

For the FRW metric of (13) one readily obtains from (17) that

$$
\frac{d}{d a}(\rho a)=-p
$$

Assuming an equation of state of the form $p=\gamma \rho$, where $\gamma$ is a constant, (19) immediately leads to

$$
\rho=C a^{-\gamma-1},
$$


where $C$ is a constant. Equation (18) is seen to be identically satisfied and does not give rise to anything new. For a pressureless (dust) pure matter universe $\left(\rho_{m} \neq 0, \rho_{r}=\right.$ $0, \gamma=0)$ we have

$$
\rho_{m}=C_{m} a^{-1}
$$

while for a pure radiation universe $\left(\rho_{m}=0, \rho_{r} \neq 0, \gamma=1\right)$, one has

$$
\rho_{r}=C_{r} a^{-2}
$$

Denoting the present time by $t_{0}$ and using the usual notation of $a_{0} \equiv a\left(t_{0}\right)$ and $\rho_{0} \equiv \rho\left(t_{0}\right)$ to denote present-day values of these quantities, we can write for a matter-dominated universe

$$
p_{m}=0, \quad \rho_{m}(t)=\rho_{m_{0}} \frac{a_{0}}{a(t)},
$$

while for a radiation-dominated universe one has

$$
p_{r}=\rho_{r}(t)=\rho_{r_{0}}\left(\frac{a_{0}}{a(t)}\right)^{2}
$$

Finally we wish to note that for the FRW metric the curvature scalar of this two-dimensional universe is given by

$$
R=-\frac{2 \ddot{a}}{a(t)},
$$

where $\ddot{a}=d^{2} a / d t^{2}$.

\section{Solutions of the Cosmological Field Equations}

In this section we seek solutions of the cosmological field equation (15) with the energy density and pressure given by (23) and (24) for each component of the cosmological fluid thus obtaining two sets of equations. For the matter dominated epoch we obtain the following:

$$
\begin{gathered}
\frac{1}{2}\left(R f^{\prime}(R)-f(R)\right)+\frac{\dot{a}}{a} \partial_{t} f^{\prime}(R)=g_{N} \rho_{m 0} \frac{a_{0}}{a}, \\
\frac{1}{2}\left(R f^{\prime}(R)-f(R)\right)+\partial_{t}^{2} f^{\prime}(R)=0 .
\end{gathered}
$$

For the radiation dominated epoch the corresponding equations read

$$
\begin{gathered}
\frac{1}{2}\left(R f^{\prime}(R)-f(R)\right)+\frac{\dot{a}}{a} \partial_{t} f^{\prime}(R)=g_{N} \rho_{r 0} \frac{a_{0}^{2}}{a^{2}}, \\
\frac{1}{2}\left(R f^{\prime}(R)-f(R)\right)+\partial_{t}^{2} f^{\prime}(R)=-g_{N} \rho_{r 0} \frac{a_{0}^{2}}{a^{2}}
\end{gathered}
$$

To proceed further we need to specify the function $f(R)$. Similar to the procedure followed in the four-dimensional case [7] we take for $f(R)$ the following expression:

$$
f(R)=R+\alpha R^{n},
$$

where the real constants $\alpha$ and $n$ are, at this stage, only restricted by $\alpha \neq 0$ and $n \neq 1$. Upon substitution of (29) into (26) we obtain

$$
\begin{gathered}
\frac{1}{2}(n-1) \alpha R^{n}+n(n-1) \alpha R^{n-2} \dot{R} \frac{\dot{a}}{a}=g_{N} \rho_{m 0} \frac{a_{0}}{a}, \\
2 n R \ddot{R}+2 n(n-2) \dot{R}^{2}+R^{3}=0 .
\end{gathered}
$$

Equations (30) and (31) describe the matter dominated epoch and we shall attempt to find solutions for them now. We start with (31) and note that in terms of the function $z(R)$ defined by

$$
z(R)=\dot{R}^{2}
$$

the equation is transformed into the following form:

$$
\frac{d z}{d R}+\frac{2(n-2)}{R} z+\frac{R^{2}}{n}=0
$$

This equation is easily solved and we obtain for $n \neq 1 / 2$

$$
z(R)=\dot{R}^{2}=-\frac{1}{n(2 n-1)} R^{3}+C_{1} R^{4-2 n},
$$

where $C_{1}$ is a constant. Equation (34) then leads to the parametric solution:

$$
t= \pm \int\left[-\frac{R^{3}}{n(2 n-1)}+C_{1} R^{4-2 n}\right]^{-1 / 2} d R+C_{2}
$$

where $C_{2}$ is a constant. For $n=2$ and $C_{1} \neq 0$ one can carry out the integration using the result [16]

$$
\int \frac{d x}{\left(K-x^{\alpha+2}\right)^{1 / 2}}=\frac{x}{\sqrt{K}}{ }_{2} F_{1}\left(\frac{1}{2}, \frac{1}{\alpha+2}, \frac{\alpha+3}{\alpha+2} ; \frac{x^{\alpha+2}}{K}\right) \text {, }
$$

where $\alpha$ and $K$ are constants and ${ }_{2} F_{1}$ is the hypergeometric function. We obtain

$$
t= \pm\left(\frac{6}{C_{1}}\right)^{1 / 2} R_{2} F_{1}\left(\frac{1}{2}, \frac{1}{3}, \frac{4}{3} ; \frac{R^{3}}{C_{1}}\right)+C_{2} .
$$

Ideally one should solve (37) to obtain $R$ as a function of the cosmic time $t$ and plug that into (30) in order to solve for $a(t)$ in the case of $n=2$, but that is a difficult task. Instead we consider solutions for which $C_{1}=0$ in (34) and a general $n \neq 1 / 2$. One can then easily derive that

$$
R=-\frac{4 n(2 n-1)}{\left(t-t_{m}\right)^{2}}
$$

where we have renamed the integration constant $C_{2}$ as $t_{m}$. In fact one can verify directly by substitution that the expression for $R$ in (38) is a solution of (31).

Next we substitute (38) into (30) and obtain

$$
A_{2} \dot{a}+A_{1}\left(t-t_{m}\right)^{-1} a=K a_{0}\left(t-t_{m}\right)^{2 n-1},
$$


where

$$
\begin{gathered}
A_{1}=\frac{1}{2}(n-1) N^{n}, \quad A_{2}=-2 n(n-1) N^{n-1}, \\
N=4 n(1-2 n), \quad K=\frac{g_{N} \rho_{m 0}}{\alpha} .
\end{gathered}
$$

We readily solve (39) and get

$$
a(t)=\bar{C}\left(t-t_{m}\right)^{1-2 n}+\bar{K}\left(t-t_{m}\right)^{2 n},
$$

where $\bar{C}$ is a constant and

$$
\bar{K}=\frac{K a_{0}}{(4 n-1) A_{2}} .
$$

Clearly $n$ must be such that $A_{1}$ and $A_{2}$ are real and $\bar{K}$ is finite. We will return to this issue later. It is interesting to note that the $t$ dependence of $R$ is $\left(t-t_{m}\right)^{-2}$ and thus independent of $n$, while that of $a(t)$ does depend on $n$. We also note that the relation $R=-2 \ddot{a} / a$ is satisfied by the solutions for $R$ given in (38) and (42), respectively. We further note that the second term in (42) is a solution of (39) in its own right. On the other hand the first term in (42) is a solution of the homogeneous form of (39). Furthermore the constants $\bar{C}$ and $\bar{K}$ must be such that $a(t)$ is positive.

We now turn to the case of radiation. Upon adding (27) and (28) we obtain

$$
R f^{\prime}(R)-f(R)+\partial_{t}^{2} f^{\prime}(R)+\frac{\dot{a}}{a} \partial_{t} f^{\prime}(R)=0 .
$$

Employing in (44) the expression for $f(R)$ given in (29) above yields

$$
n R \ddot{R}+n(n-2) \dot{R}^{2}+R^{3}+n R \dot{R} \frac{\dot{a}}{a}=0 .
$$

Next we use (29) in (27) and obtain

$$
\frac{1}{2}(n-1) \alpha R^{n}+n(n-1) \alpha R^{n-2} \dot{R} \frac{\dot{a}}{a}=g_{N} \rho_{r 0} \frac{a_{0}^{2}}{a^{2}} .
$$

Motivated by the structure of the solutions for the cosmological equations in the case of pure matter above, we seek solutions for $R(t)$ and $a(t)$ of (45) and (46) in the form of powers in $t-t_{r}$ where $t_{r}$ is some reference time. We obtain the following results:

$$
\begin{gathered}
R(t)=2 n(n-1)\left(t-t_{r}\right)^{-2}, \\
a(t)=B\left(t-t_{r}\right)^{n},
\end{gathered}
$$

where the constant $B$ is given by

$$
B=\left[\frac{g_{N} \rho_{r 0}}{n(n-1)(1-3 n)[2 n(1-n)]^{n-1} \alpha}\right]^{1 / 2} a_{0} .
$$

Note that, as in the case of matter, the $t$ dependence of $R(t)$ is independent of $n$, the only such dependence appears in the overall coefficient. We also note that the relation $R=-2 \ddot{a} / a$ is satisfied by the solutions for $R$ given in (46) and (48). For an expanding universe one must have $n>1$ and $B>0$. Furthermore the value of $n$ must ensure that the bracketed term in (49) is finite and real.

\section{Properties of the Solutions}

We now discuss some properties of the solutions of the cosmological field equations found in the previous section. Let us first look at the radiation dominated case and determine whether our vision of the universe is limited by a particle horizon. At a given cosmic time $t_{s}$ the proper distance $d\left(t_{s}\right)$ of the emitter is given by

$$
d\left(t_{s}\right)=a\left(t_{s}\right) \int_{t_{e}}^{t_{s}} \frac{d t^{\prime}}{a\left(t^{\prime}\right)},
$$

where $t_{e}$ is the time of emission of the photon. Using (48) we obtain

$$
d\left(t_{s}\right)=\frac{\left(t_{s}-t_{r}\right)^{n}}{1-n}\left[\left(t_{s}-t_{r}\right)^{1-n}-\left(t_{e}-t_{r}\right)^{1-n}\right] .
$$

We can view $t_{r}$ as signifying the onset of the radiation epoch. We see that as $t_{e} \rightarrow t_{r}, d\left(t_{s}\right)$ is finite for $1-n>0$ and diverges for $1-n<0$. Hence no particle horizon problem will arise if $n>1$ which is the same condition required for an expanding universe. Reality of $B$ also requires $n$ to be an integer. For $n$ an even integer, the parameter $\alpha$ must be positive while for $n$ odd, $\alpha$ should be negative. Thus we take $n$ to be a positive integer greater than one. Next we note that the cosmic acceleration $\ddot{a}(t)$ which is given by

$$
\ddot{a}(t)=n(n-1) B\left(t-t_{r}\right)^{n-2},
$$

is positive for $t>t_{r}$ since $n>1$ and is constant for $n=2$. Now in two-dimensional spacetime the radiation energy density is $\rho_{r} \propto T^{2}$ where $T$ is the temperature [16] and it follows therefore from (24) that

$$
a \propto T^{-1} .
$$

Since we have $a \rightarrow 0$ as $t \rightarrow t_{r}$, we conclude that this radiation universe has a hot big bang origin.

Next we turn to the case of the matter dominated universe described by (38) and (42). First let us consider the case $\bar{C}=0$ when the scale factor becomes

$$
\bar{a}(t)=\bar{K}\left(t-t_{m}\right)^{2 n} .
$$

As we have stated earlier this is viable because it represents a solution of (39). The time $t_{m}$ can be taken to signify the onset of matter dominance. The proper distance $d\left(t_{s}\right)$ is now given by

$$
d\left(t_{s}\right)=\frac{\left(t_{s}-t_{m}\right)^{2 n}}{1-2 n}\left[\left(t_{s}-t_{m}\right)^{1-2 n}-\left(t_{e}-t_{m}\right)^{1-2 n}\right] .
$$

Hence no particle horizon will arise if $2 n>1$. Also as we stated following (43), the parameter $n$ must be such that the constants $A_{1}$ and $A_{2}$ given by (40) are real. Since for $2 n>1$ the number $N$ of (41) is negative, it follows that $n$ has to be a positive integer. Now the requirement that $\bar{a}(t)>0$ for $t>t_{m}$ implies that $\bar{K}>0$. For $n$ even we have $A_{2}>0$ and hence $\alpha$ should be positive to ensure $\bar{K}>0$ while for $n$ odd one has $A_{2}<0$ and $\alpha$ should be negative. Since we exclude $n=1$, the smallest permissible value is $n=2$. For such values of 
$n$ it is evident that the cosmic acceleration $\ddot{\bar{a}}(t)$ is positive. Finally we observe that for the pure matter universe we have $\bar{a}(t) \rightarrow 0$ as $t \rightarrow t_{m}$.

We now consider the case $C \neq 0$. Using (42) the proper distance is now given by

$$
d\left(t_{s}\right)=a\left(t_{s}\right) \int_{t_{e}}^{t_{s}} \frac{\left(t-t_{m}\right)^{2 n-1}}{C+\bar{K}\left(t-t_{m}\right)^{4 n-1}} d t .
$$

It is clear that the integral converges for $t_{e} \rightarrow t_{m}$ and we do have a particle horizon. Performing the integral we determine the proper distance to the horizon to be

$$
\begin{aligned}
d\left(t_{s}\right)= & \frac{a\left(t_{s}\right)}{\bar{C}}\left(\frac{\bar{K}}{\bar{C}}\right)^{2 n(4 n-1)} \\
& \times\left\{-\frac{\ln \left(1+\xi_{s}\right)}{4 n-1}-\frac{1}{4 n-1} \sum_{k=1}^{2 n-1} \cos \left[\frac{2 n \pi(2 k-1)}{4 n-1}\right]\right. \\
& \times \ln \left(1-2 \xi_{s} \cos \frac{2 k-1}{4 n-1} \pi+\xi_{s}^{2}\right) \\
& +\frac{2}{4 n-1} \sum_{k=1}^{2 n-1} \sin \left[\frac{2 n \pi(2 k-1)}{4 n-1}\right] \\
& \times \operatorname{arctg}\left[\frac{\xi_{s}-\cos ((2 k-1) /(4 n-1)) \pi}{\sin ((2 k-1) /(4 n-1)) \pi}\right] \\
& \left.-\left(\xi_{s} \longleftrightarrow \xi_{e}\right)\right\}
\end{aligned}
$$

where

$$
\xi_{j}=\left(\frac{\bar{C}}{\bar{K}}\right)^{4 n-1}\left(t_{j}-t_{m}\right), \quad j=s, e .
$$

Let us now study further properties of the solution given in (42). In the following we consider only values of $t$ such that $t>t_{m}$. Now it is evident that, except for values of $n$ in the interval $0<n<1 / 2$, the first term in (42) dominates for $t$ near $t_{m}$ when $n>1 / 2$ while the second term dominates for $n<0$. Hence to ensure positivity of the scale factor we require that both $\bar{C}$ and $\bar{K}$ be positive. For $0<n<1 / 2, \bar{C}$ and $\bar{K}$ can have opposite signs but only in such a manner so as to keep $a>0$. We shall for simplicity assume that $\bar{C}>0$ and $\bar{K}>0$ for all values of $n$. Next we observe that outside the interval $0<n<1 / 2$, the number $N$ of (41) is negative, and to ensure the reality of $A_{2}$ given by (40), the number $n$ has to be an integer. We readily deduce that for $\alpha>0, n$ can be a positive even integer or a negative odd integer. On the other hand for $\alpha<0, n$ can be a positive odd integer or a negative even integer. The cosmic acceleration $\ddot{a}(t)$ is given by

$$
\ddot{a}(t)=2 n(2 n-1)\left(t-t_{m}\right)^{-2} a(t) .
$$

It is seen that $\ddot{a}<0$ for $0<n<1 / 2, \ddot{a}=0$ for $n=1 / 2$, and $\ddot{a}>0$ for $n<0$ or $n>1 / 2$.

Next we consider the behavior of $a(t)$ as $t \rightarrow t_{m}$ for the case $\bar{C} \neq 0$. We see from (42) that for $0<n<1 / 2, a(t) \rightarrow 0$ as $t \rightarrow t_{m}$ and accordingly the temperature $T \rightarrow \infty$ in this limit. For $n=1 / 2$, we have $a(t) \rightarrow \bar{C}$ as $t \rightarrow t_{m}$ and $T$ is finite. However for $n$ outside the interval $0 \leq n \leq 1 / 2$ the behavior of $a(t)$ is very different as $t \rightarrow t_{m}$. We see that $a(t) \rightarrow \infty$ in this limit and energy density $\rho_{m}$ and the temperature tend to zero. As $t$ increases beyond the value $t_{m}, a(t)$ decreases to finite values and the density increases. However $a(t)$ never reaches zero and attains a minimum value at $t=t_{c}$ given by

$$
t_{c}=t_{m}+\left[\frac{(2 n-1) \bar{C}}{2 n \bar{K}}\right]^{1 / 4 n-1} .
$$

For $t>t_{c}, a(t)$ starts to increase. We also note from (38) that the curvature scalar is $R \rightarrow-\infty$ as $t \rightarrow t_{m}$ and then starts increasing through finite negative values as $t$ grows beyond $t_{m}$. The singular behavior of the scale factor noted here should be contrasted with that of the FRW cosmological models in four-dimensional general relativity where the scale factor and energy density go to zero and infinity, respectively, as the initial moment is approached.

\section{Inflation}

The horizon problem in four-dimensional standard FRW cosmology is a consequence of deceleration in the expansion of the universe. The problem can be solved by postulating a phase of the universe, prior to the decelerating phase, in which the expansion is accelerating and such a phase is called a period of inflation. Hence inflation is characterized by the following property for the scale factor $a(t)$ :

$$
\ddot{a}(t)>0 .
$$

Now as evident from the analysis of Section $4, \ddot{a}>0$ is readily achieved in our $f(R)$ theory in two-dimensional spacetime and the universe is accelerating. The solutions obtained for the scale factor displayed power dependence on time akin to that of power-law inflation. It would seem that there is no need to require an inflationary phase since matter or radiation dominated epochs yield an accelerating universe. Here we are not seeking to introduce scalar fields to propel acceleration as in the usual inflationary cosmology. We recall that one of the motivations for introducing modified or $f(R)$ theories of gravity in four-dimensional spacetime is the desire to explain acceleration of the universe as an alternative to using scalar fields. For this purpose solutions for the cosmological field equations are sought in the absence of the matter fluid [7]. We carry out such an analysis in our case by considering solutions to (30) and (31) of Section 3 with the R.H.S set being equal to zero. We have earlier obtained a general solution for (31) given by (35) of Section 3. However the parametric nature of that solution makes it difficult to use in (30) in order to solve for $a(t)$. Putting $C_{1}=0$ enables the integration in (35) to be performed and leads to the solution given in (38) which we write as

$$
R=-\frac{4 n(2 n-1)}{(t-\bar{t})^{2}}
$$


where $n \neq 1 / 2,1$ and we have now denoted the integration constant by $\bar{t}$. Using (62) in (30) with the R.H.S. set being equal to zero yields:

$$
\dot{a}+\frac{2 n-1}{t-\bar{t}} a=0,
$$

the solution of which reads

$$
a(t)=A(t-\bar{t})^{1-2 n},
$$

where $A>0$ is a constant. We take the solution to hold for $t>\bar{t}$. The cosmic acceleration is given by

$$
\ddot{a}(t)=2 n(2 n-1) A(t-\bar{t})^{-2 n-1} .
$$

The Hubble parameter is

$$
\begin{gathered}
H=\frac{\dot{a}}{a}=\frac{1-2 n}{t-\bar{t}}, \\
\dot{H}=\frac{2 n-1}{(t-\bar{t})^{2}} .
\end{gathered}
$$

For $n<-1$ we can identify $\bar{t}$ with the onset of inflation $\bar{t}=t_{i}$. Equation (64) then describes a universe that expands with positive acceleration for $t>t_{i}$. We also have $H>0$ and $\dot{H}<0$ for $t>t_{i}$ which characterizes standard inflation. However if we make the identification $\bar{t}=t_{i}$ for $n>1$, we will have a situation in which $a(t) \rightarrow \infty$ as $t \rightarrow t_{i}$ thus obtaining a universe that starts off already with an infinite size at the onset of inflation collapsing subsequently for $t>t_{i}$ at an accelerated rate. Such a scenario can be avoided if $\bar{t}$ is instead taken to have a relatively large value so that $t<\bar{t}$ during the inflationary epoch. We write $a(t)$ now as

$$
a(t)=A|t-\bar{t}|^{1-2 n} .
$$

The universe then starts off with a relatively small non-zero size at $t=t_{i}$ and expands with positive acceleration as time progresses. We also have

$$
\begin{aligned}
H & =\frac{2 n-1}{\bar{t}-t}, \\
\dot{H} & =\frac{1-2 n}{(\bar{t}-t)^{2}},
\end{aligned}
$$

so that $H>0$ and $\dot{H}<0$ and we again have standard inflation.

As in four spacetime dimensions we define the so-called slow-roll parameter $\varepsilon$ by [7]

$$
\varepsilon=-\frac{\dot{H}}{H^{2}} .
$$

and in terms of which one has

$$
\frac{\ddot{a}}{a}=H^{2}+\dot{H}=(1-\varepsilon) H^{2} .
$$

Inflation can thus be attained only if $\varepsilon<1$. In our present context $\varepsilon$ is given by

$$
\varepsilon=\frac{1}{|2 n-1|}
$$

For both cases of $n<-1$ and $n>1$ we clearly have $\varepsilon<1$. The slow-roll approximation corresponding to $\varepsilon \ll 1$ then obtain when $|2 n-1| \gg 1$. As we have stated previously the solution for $R$ given in (62) arises as a special case of the general solution given in (35). As an alternative to solving (30) and (31) one can derive an equation for the Hubble parameter [7, 17]. We write (30) with the R.H.S set being equal to zero:

$$
2 n \dot{R} \dot{a}+R^{2} a=0 .
$$

Now from (25) of Section 2 we obtain

$$
\dot{R}=-\frac{2 \dddot{a}}{a}+\frac{2 \ddot{a} \dot{a}}{a^{2}} .
$$

Substituting (25) and (73) in (72) one obtains

$$
-n a \ddot{a} \dddot{a}+a \ddot{a}^{2}+n \dot{a}^{2} \ddot{a}=0 .
$$

Next in terms of $H, \dot{H}$, and $\ddot{H}$ we can express (74), after some manipulations, as

$$
-n H \ddot{H}-2(n-1) \dot{H} H^{2}+\dot{H}^{2}+H^{4}=0 .
$$

It is customary, in dealing with equations such as this, to invoke the slow-roll approximation $\left|\dot{H} / H^{2}\right| \ll 1$ and $|\ddot{H} / H \dot{H}| \ll 1,[7,17]$. Applying this to $(67)$ we obtain that

$$
-2(n-1) \dot{H}+H^{2}=0 .
$$

The solution of (76) is

$$
H(t)=\frac{-2(n-1)}{t-\bar{t}^{\prime}},
$$

where $\bar{t}^{\prime}$ is a constant. Equation (77) in turn gives

$$
a(t)=\frac{A^{\prime}}{\left(t-\bar{t}^{\prime}\right)^{2(n-1)}}
$$

with $A^{\prime}$ being another constant. Equation (78) for $a(t)$ is similar in structure to (67) and the properties of the solution are therefore similar to what we discussed before and hence will not be considered any further.

We shall next seek a general solution to (31) for $R(t)$ that holds for $t$ close to the instant $t_{i}$ that signifies the onset of inflation. Specifically we assume that $t=t_{i}$ is a regular point of (31) and seek a solution for $R(t)$ in the form of a power series confining ourselves to small values of $t-t_{i}$. For simplicity we consider the case $n=2$ for which (31) becomes

$$
4 \ddot{R}+R^{2}=0 .
$$

We write

$$
R(t)=\sum_{m=0}^{\infty} b_{m}\left(t-t_{i}\right)^{m} .
$$

Substituting (80) in (79) and solving we obtain

$$
\begin{gathered}
b_{2}=-\frac{1}{8} b_{0}^{2}, \\
b_{3}=-\frac{1}{12} b_{0} b_{1},
\end{gathered}
$$


and so forth. This leads to

$$
\begin{aligned}
R(t)= & b_{0}+b_{1}\left(t-t_{i}\right)-\frac{1}{8} b_{0}^{2}\left(t-t_{i}\right)^{2} \\
& -\frac{1}{12} b_{0} b_{1}\left(t-t_{i}\right)^{3}+\cdots .
\end{aligned}
$$

We remark that if inflation lasts for a short period of time, then it is sensible to have a representation for $R(t)$ as given in (82). Moreover for sufficiently small $t-t_{i}$ we can approximate $R(t)$ by the first two terms and substitute in (72) with $n=2$. Solving the resulting equation we obtain

$$
a(t) \approx C \exp \left\{-\frac{1}{12 b_{1}^{2}}\left[b_{0}+b_{1}\left(t-t_{i}\right)\right]^{3}\right\},
$$

where $C>0$ is a constant. We can write $(83)$ as

$$
a(t) \approx a_{i} \exp \left\{-\frac{1}{12 b_{1}^{2}}\left(\left[b_{0}+b_{1}\left(t-t_{i}\right)\right]^{3}-b_{0}^{3}\right)\right\},
$$

where

$$
a_{i}=a\left(t_{i}\right)=C \exp \left(-\frac{b_{0}^{3}}{12 b_{1}^{2}}\right) .
$$

From (83) we obtain

$$
\begin{gathered}
\dot{a}(t)=-\frac{1}{4 b_{1}}\left[b_{0}+b_{1}\left(t-t_{i}\right)\right]^{2} a(t), \\
\ddot{a}(t)=\left\{-\frac{1}{2}\left[b_{0}+b_{1}\left(t-t_{i}\right)\right]+\frac{1}{16 b_{1}^{2}}\left[b_{0}+b_{1}\left(t-t_{i}\right)\right]^{4}\right\} a(t) .
\end{gathered}
$$

From (84) we see that we must have $b_{1}<0$ to ensure that $\dot{a}>0$. We must also require $a(t)$ to be increasing for $t>t_{i}$. This can be achieved by having $b_{0}>0$ for then $b_{0}+b_{1}\left(t-t_{i}\right)$ will start off at the value $b_{0}$ and decreases reaching zero at $t^{*}-t_{i}=-b_{0} / b_{1}$. During the interval, $t_{i}<t<t^{*}, a(t)$ will be increasing. We must also require the cosmic acceleration $\ddot{a}(t)$ to be positive during this interval and this leads to the following condition:

$$
\frac{1}{8 b_{1}^{2}}\left[b_{0}+b_{1}\left(t-t_{i}\right)\right]^{3}>1
$$

This inequality will continue to hold until $t=t_{f}<t^{*}$ when $\ddot{a}\left(t_{f}\right)=0$. This implies that

$$
\frac{1}{8 b_{1}^{2}}\left[b_{0}+b_{1}\left(t_{f}-t_{i}\right)\right]^{3}=1
$$

which yields

$$
t_{f}=t_{i}+b_{0}\left|b_{1}\right|^{-1}-2\left|b_{1}\right|^{-1 / 3} .
$$

The time $t_{f}$ then signifies the end of inflation. Since $R\left(t_{i}\right)=$ $b_{0}$ and $\dot{R}\left(t_{i}\right)=b_{1}$, the conditions $b_{0}>0$ and $b_{1}<0$ can be expressed as

$$
\begin{aligned}
& R\left(t_{i}\right)>0, \\
& \dot{R}\left(t_{i}\right)<0 .
\end{aligned}
$$

We can also express the duration of inflation as

$$
t_{f}-t_{i}=R\left(t_{i}\right)\left|\dot{R}\left(t_{i}\right)\right|^{-1}-2\left|\dot{R}\left(t_{i}\right)\right|^{-1 / 3} .
$$

The Hubble parameter is given by

$$
H=-\frac{1}{4 b_{1}}\left[b_{0}+b_{1}\left(t-t_{1}\right)\right]^{2}
$$

It thus decreases from an initial value $H_{i}$ given by

$$
H_{i}=H\left(t_{i}\right)=-\frac{b_{0}^{2}}{4 b_{1}}=\frac{R^{2}\left(t_{i}\right)}{4\left|\dot{R}\left(t_{i}\right)\right|},
$$

to a value $H_{f}$ at the end of inflation where

$$
H_{f}=H\left(t_{f}\right)=\left|b_{1}\right|^{1 / 3}=\left|\dot{R}\left(t_{i}\right)\right|^{1 / 3} .
$$

We note that

$$
\dot{H}=-\frac{1}{2}\left[b_{0}+b_{1}\left(t-t_{i}\right)\right]
$$

is negative during $t_{i}<t<t_{f}$ and we thus have standard inflation. The slow-roll parameter is given by

$$
\varepsilon=8 b_{1}^{2}\left[b_{0}+b_{1}\left(t-t_{i}\right)\right]^{-3} .
$$

We recall that for inflation to proceed one must have $\varepsilon<1$ and this leads precisely to the condition expressed in (87) stated earlier.

The number of $e$-foldings from $t=t_{i}$ to $t=t_{f}$ is defined by $[7,18]$

$$
N=\int_{t_{i}}^{t_{f}} H d t
$$

which is evaluated to give

$$
N=\frac{2}{3}\left[\left(\frac{H_{i}}{H_{f}}\right)^{3 / 2}-1\right]
$$

In four dimensions, the solution of the horizon and flatness problems of big bang cosmology requires that $N \geq 70$, $[7,19]$. If we assume that we can use this value in our twodimensional universe, we find that

$$
\frac{H_{i}}{H_{f}} \geq 22
$$

that is, the Hubble parameter decreases to about $4.5 \%$ of its initial value by the time inflation ends.

\section{Quantization}

As we stated in the introduction two-dimensional spacetime models of gravity provide an arena where issues like quantization are studied since in such a setting they prove to be more tractable than in four-dimensional spacetime. In this section we thus consider quantization of the $f(R)$ gravity theory defined by the action of (3). Our objective is to derive the Wheeler-DeWitt equation for the wave function of the 
universe and obtain its solutions. Since we are considering a spatially homogeneous and isotropic universe, we drop the spatial integral and write the action as

$$
I_{G}=-\frac{1}{2 g_{N}} \int d t a(t) f(R(t)) .
$$

We take for $f(R)$ the expression given in (29) and put $n=2$. We use (25) that expresses the scalar curvature in terms of the scale factor and write

$$
I_{G}=-\frac{1}{2 g_{N}} \int d t(a R-2 \alpha \ddot{a} R) .
$$

We notice the appearance of the second derivative of $a$ in (101). The standard approach is to express the wave function in terms of $a$ and $R[20]$. Hence integrating by parts in (101), we obtain

$$
I_{G}=\int \mathcal{L}(a, \dot{a}, k, \dot{k}) d t
$$

where

$$
\mathcal{L}=-\frac{1}{2 g_{N}}(a R+2 \alpha \dot{a} \dot{R})
$$

The canonical momenta are defined in the usual way:

$$
\begin{aligned}
& P_{a}=\frac{\partial \mathcal{L}}{\partial \dot{a}}=-\frac{\alpha}{g_{N}} \dot{R} \\
& P_{R}=\frac{\partial \mathcal{L}}{\partial \dot{R}}=-\frac{\alpha}{g_{N}} \dot{a} .
\end{aligned}
$$

The Hamiltonian is then obtained as

$$
\mathscr{H}=P_{a} \dot{a}+P_{R} \dot{R}-\mathcal{L}=-\frac{g_{N}}{\alpha} P_{a} P_{R}+\frac{1}{2 g_{N}} a R .
$$

Replacing $P_{a}$ and $P_{R}$ by $-i(\partial / \partial a)$ and $-i(\partial / \partial R)$, respectively, in the Hamiltonian, we obtain the Wheeler-DeWitt equation for the wave function of the universe:

$$
\left(\frac{g_{N}}{\alpha} \frac{\partial^{2}}{\partial R \partial a}+\frac{1}{2 g_{N}} a R\right) \psi(a, R)=0
$$

Instead of $a$ and $R$ we shall work with the variables:

$$
\xi=R+a, \quad \eta=R-a .
$$

In terms of $\xi$ and $\eta$ the Wheeler-DeWitt equation becomes

$$
\left[\frac{g_{N}}{\alpha}\left(\frac{\partial^{2}}{\partial \xi^{2}}-\frac{\partial^{2}}{\partial \eta^{2}}\right)+\frac{1}{g_{N}}\left(\xi^{2}-\eta^{2}\right)\right] \psi(\xi, \eta)=0 .
$$

We seek solutions of (108) in factorizable form:

$$
\psi(\xi, \eta)=X(\xi) Y(\eta)
$$

and obtain the following equations for the functions $X$ and Y:

$$
\begin{aligned}
& \frac{d^{2} X}{d \xi^{2}}+\frac{\alpha}{8 g_{N}^{2}} \xi^{2} X=\frac{C \alpha}{g_{N}} X \\
& \frac{d^{2} Y}{d \eta^{2}}+\frac{\alpha}{8 g_{N}^{2}} \eta^{2} Y=\frac{C \alpha}{g_{N}} Y
\end{aligned}
$$

where $C$ is the separation constant. The two equations are identical and hence it is enough to consider one of them. We first take $\alpha>0$ and define

$$
\begin{aligned}
& \gamma^{2}=\frac{\alpha}{8 g_{N}^{2}}, \\
& E=-\frac{C \alpha}{2 g_{N}} .
\end{aligned}
$$

In terms of $\gamma^{2}$ and $E$, (110) reads

$$
\frac{d^{2} X}{d \xi^{2}}+\gamma^{2} \xi^{2} X+2 E X=0
$$

It is interesting to note that (114) is identical to that describing the inverted or reversed oscillator discussed by several authors in a number of contexts [21-25]. By performing the change of variable

$$
y=\sqrt{2 \gamma} \xi
$$

we cast (114) into the following form:

$$
\frac{d^{2} X}{d y^{2}}+\frac{1}{4} y^{2} X+\varepsilon X=0,
$$

where $\varepsilon=E / \gamma$. Equation (116) is one of the standard forms of the equation for the parabolic cylinder functions. Two linearly independent solutions are given by the real functions $W(\varepsilon, y)$ and $W(\varepsilon,-y)[26]$. For $|y| \gg 1$ and $|y| \gg|\varepsilon|$ these solutions display the following asymptotic behaviour:

$$
\begin{gathered}
W(\varepsilon, y \longrightarrow \infty) \sim \sqrt{\frac{2 k}{y} \cos \left(\frac{1}{4} y^{2}+\varepsilon \ln y+\frac{1}{4} \pi+\frac{1}{2} \phi\right),} \\
W(\varepsilon, y \longrightarrow-\infty) \sim \sqrt{\frac{2}{k|y|}} \sin \left(\frac{1}{4} y^{2}+\varepsilon \ln |y|+\frac{1}{4} \pi+\frac{1}{2} \phi\right),
\end{gathered}
$$

where

$$
\begin{gathered}
k=\left(1+e^{-2 \pi \varepsilon}\right)^{1 / 2}-e^{-\pi \varepsilon}, \\
\phi=\arg \Gamma\left(\frac{1}{2}-i \varepsilon\right) .
\end{gathered}
$$

The functions $W(\varepsilon, y)$ and $W(\varepsilon,-y)$ satisfy the following normalization conditions [23]:

$$
\begin{gathered}
\int_{-\infty}^{\infty} W(\varepsilon, y) W\left(\varepsilon^{\prime},-y\right) d y= \begin{cases}0 & \text { if } \varepsilon \neq \varepsilon^{\prime} \\
\frac{\pi e^{-\pi \varepsilon}}{\left(1+e^{-2 \pi \varepsilon}\right)^{1 / 2}} & \text { if } \varepsilon=\varepsilon^{\prime}\end{cases} \\
\int_{-\infty}^{\infty} W(\varepsilon, y) W\left(\varepsilon^{\prime}, y\right) d y=2 \pi\left(1+e^{-2 \pi \varepsilon}\right)^{1 / 2} \delta\left(\varepsilon-\varepsilon^{\prime}\right) .
\end{gathered}
$$

The parabolic cylinder functions can be expressed in several forms [26] and we can use the various relations between these forms to express $W(a, x)$ in terms of the more familiar function $D_{p}(x)$ for some $p$. In fact one can easily derive that

$$
\begin{aligned}
W(\varepsilon, y)=\left(\frac{k}{2}\right)^{1 / 2}[ & e^{i \theta} D_{i \varepsilon-1 / 2}\left(y e^{-(i / 4) \pi}\right) \\
& \left.+e^{-i \theta} D_{-i \varepsilon-1 / 2}\left(y e^{(i / 4) \pi}\right)\right]
\end{aligned}
$$


where

$$
\theta=\frac{1}{2}\left(-\frac{1}{2} \pi \varepsilon+\frac{i}{4} \pi+i \phi\right) .
$$

Next we observe that the solutions to (111) are identical to those of (110) but expressed in terms of the vriable $\eta$. Hence we can write the following for the wavefunction $\psi$ :

$$
\psi(\xi, \eta)=\psi_{1}(\xi) \psi_{2}(\eta),
$$

where

$$
\begin{aligned}
& \psi_{1}(\xi)=C_{1} W\left(\frac{E}{\gamma}, \sqrt{2 \gamma} \xi\right)+C_{2} W\left(\frac{E}{\gamma},-\sqrt{2 \gamma} \xi\right), \\
& \psi_{2}(\xi)=C_{1}^{\prime} W\left(\frac{E}{\gamma}, \sqrt{2 \gamma} \eta\right)+C_{2}^{\prime} W\left(\frac{E}{\gamma},-\sqrt{2 \gamma} \eta\right) .
\end{aligned}
$$

We now consider the case in which the parameter $\alpha$ is negative and write (110) and (111) as

$$
\begin{aligned}
& \frac{d^{2} X}{d \xi^{2}}-\frac{|\alpha|}{8 g_{N}^{2}} \xi^{2} X=-\frac{C|\alpha|}{g_{N}} X, \\
& \frac{d^{2} Y}{d \eta^{2}}-\frac{|\alpha|}{8 g_{N}^{2}} \eta^{2} Y=-\frac{C|\alpha|}{g_{N}} Y .
\end{aligned}
$$

We define

$$
\begin{gathered}
\gamma^{2}=\frac{|\alpha|}{8 g_{N}^{2}}, \\
E=-\frac{C|\alpha|}{2 g_{N}},
\end{gathered}
$$

and thus they retain the same forms as in (112) and (113), respectively. Focussing on (124) we write it as

$$
\frac{d^{2} X}{d \xi^{2}}-\gamma^{2} \xi^{2} X=2 E X
$$

In terms of $y=\sqrt{2 \gamma} \xi$, (127) becomes

$$
\frac{d^{2} X}{d y^{2}}+\left(\sigma+\frac{1}{2}-\frac{1}{4} y^{2}\right) X=0
$$

where

$$
\sigma+\frac{1}{2}=-\frac{E}{\gamma}
$$

Equation (128) has the form of Weber's equation [27] and possesses the following solution:

$$
X_{1}(y)=D_{\sigma}(y)=2^{\sigma / 2+1 / 4} y^{-1 / 2} W_{\sigma / 2+1 / 4,-1 / 4}\left(\frac{y^{2}}{2}\right) .
$$

In the above equation $W_{\mu, \nu}$ is the Whittaker function. Expressing $W_{\mu, \nu}$ in terms of the confluent hypergeometric function, we can write

$$
\begin{aligned}
X_{1}(y)= & \frac{\Gamma(1 / 2) 2^{\sigma / 2}}{\Gamma(1 / 2-\sigma / 2)} e^{-y^{2} / 4} F\left(-\frac{\sigma}{2}, \frac{1}{2}, \frac{y^{2}}{2}\right) \\
& +\frac{\Gamma(-1 / 2) 2^{\sigma / 2-1 / 2}}{\Gamma(-\sigma / 2)} y e^{-y^{2} / 4} F\left(\frac{1-\sigma}{2}, \frac{3}{2}, \frac{y^{2}}{2}\right) .
\end{aligned}
$$

For the second solution of (128) we note that from (130) giving the relationship between $D_{\sigma}$ and the Whittaker function, we know that $D_{-\sigma-1}( \pm i y)$ are solutions linearly independent of $D_{\sigma}(y)$ as $W_{-(\sigma / 2)-(1 / 4),-1 / 4}\left(-y^{2} / 2\right)$ is linearly independent of $W_{(\sigma / 2)+(1 / 4),(-1 / 4)}\left(y^{2} / 2\right)$. From the asymptotic behaviour of the confluent hypergeometric function, we deduce that as $y \rightarrow \infty$,

$$
X_{1}(y) \sim e^{-y^{2} / 4} y^{\sigma}
$$

that is, $X_{1} \rightarrow 0$. For $y \rightarrow-\infty$ we have

$$
X_{1}(y) \sim-\frac{(2 \pi)^{1 / 2}}{\Gamma(-\sigma)} e^{\sigma \pi i} e^{y^{2} / 4} y^{-\sigma-1},
$$

that is, $X_{1} \rightarrow \infty$ unless $\sigma$ is a positive integer or zero in which case the R.H.S of (133) vanishes. In fact we have the relationship

$$
D_{n}(y)=2^{-n / 2} e^{-y^{2} / 4} H_{n}\left(\frac{y}{\sqrt{2}}\right), \quad n=0,1,2, \ldots
$$

that expresses the parabolic cylinder functions $D_{n}$ in terms of the Hermite polynomials $H_{n}$. Going back to (126) and (129) with $\sigma=n$, we obtain

$$
C_{n}=\frac{n+1 / 2}{\sqrt{2}|\alpha|^{1 / 2}}
$$

as the value of the separation constant. The functions $X_{n}(\xi)$ that solve (127) are then precisely those that describe the onedimensional quantum oscillator. We write

$$
X_{n}(\xi)=\left(\frac{\gamma}{\pi}\right)^{1 / 4} \frac{1}{\sqrt{2^{n} n !}} H_{n}(\sqrt{\gamma} \xi) e^{-(1 / 2) \gamma \xi^{2}}
$$

The solutions $Y_{n}(\eta)$ are identical in form and we obtain for the normalized wave function the following:

$$
\psi_{n}(\xi, \eta)=\left(\frac{\gamma}{\pi}\right)^{1 / 2} \frac{1}{2^{n} n !} H_{n}(\sqrt{\gamma} \xi) H_{n}(\sqrt{\gamma} \eta) e^{-(1 / 2) \gamma\left(\xi^{2}+\eta^{2}\right)}
$$

For $\sigma \neq n$ the wavefunctions will not have finite norm and solutions of (124) and (125) of the type given in (131) would have to be superposed, just as wave packets are constructed in quantum mechanics, in order to obtain wave functions capable of describing physical states.

\section{Conclusions}

In this work we studied $f(R)$ theories of gravity in twodimensional spacetime with focus on applications to cosmology. With the metric taken to have to the FRW form we were able to obtain solutions for the cosmological field equations in the case of pure matter or radiation-dominated universe when $f(R)=R+\alpha R^{n}$. The remarkable feature of these solutions is that they readily describe an accelerating universe in contrast to the standard FRW cosmology of four-dimensional general relativity. The horizon problem is also readily solved. As we have stated in Section 2, the 
time evolution of the scale factor is not affected by the value of the curvature constant $k$. We have also seen that the solution for the radiation-dominated universe and one solution for the case of pure matter-domination, describe a hot big bang. However an interesting solution in a matter dominated universe, given in (42), describes a universe that kicks off with an infinite size and zero temperature at the start of matter dominance. It subsequently collapses to a finite size and then begins to expand.

Now as we mentioned before, the interest behind the pursuit of $f(R)$ theories is partially due to the desire to obtain a description of inflation without the introduction of scalar fields. This is done by seeking solutions to the cosmological field equations with the energy-momentum tensor set equal to zero, [7]. In Section 5 we obtained such solutions that characterize power law inflation. Furthermore, with inflation presumed to last for a short period of time, we obtained for the case $n=2$ a solution for $t$ near $t_{i}$, the instant of onset of inflation. This solution displayed exponential dependence on time. For this case we computed the duration of inflation and the number of $e$-foldings as well as an estimate for the change that ensues in the value of the Hubble parameter from the start to the end of inflation. The basic distinguishing feature between power law and exponential inflation appears to be in the behavior of the Ricci scalar. Exponential inflation is obtained when we assumed that $R(t)$ can be expanded in a power series about $t=t_{i}$ with finite coefficients. In particular $R\left(t_{i}\right)$ and $\dot{R}\left(t_{i}\right)$ are finite. On the other hand in the case of power law inflation these quantities exhibit singular behavior at $t=\bar{t}$. Another characteristic of our inflationary solutions is that they do not depend on the parameter that appears in (29) for $f(R)$. This is in contrast to the inflationary solution in four-dimensional $f(R)$ theories where $n=2$ describes the Starobinsky model [28]. In that case with $\alpha$ being written as $\alpha=1 / 6 M^{2}$, where the constant $M$ has the dimension of mass, exponential inflation is obtained with $a, H$, and $R$ all depending on $M$, [7].

Interest in two-dimensional theories stems partially from the desire to investigate the quantum theory in a simple setting. Hence we carried out quantization of the theory in the case of $n=2$. The Wheeler-DeWitt equation was derived and its solutions were obtained. We were able to solve the equation exactly in the entire domain of the variables, unlike the situation in the four-dimensional case $[17,20]$. Interestingly we found that for $\alpha>0$ the equation for the wave function coincided with that of the inverted oscillator. For $\alpha<0$ the wave function, under certain conditions, turned out to be a product of two quantum harmonic oscillator wave functions in the variables $\xi=$ $R+a$ and $\eta=R-a$. In conclusion we have studied some aspects of classical and quantum cosmology in twodimensional $f(R)$ theories. Clearly a lot more issues need to be investigated and we hope to return to them in the near future.

\section{References}

[1] H. Weyl, Annalen der Physik, vol. 364, no. 10, pp. 101-133, 1919.
[2] A. S. Eddington, The Mathematical Theory of Relativity, Cambridge University Press, Cambridge, UK, 1923.

[3] R. Utiyama and B. S. DeWitt, "Renormalization of a classical gravitational field interacting with quantized matter fields," Journal of Mathematical Physics, vol. 3, no. 4, pp. 608-618, 1962.

[4] K. S. Stelle, "Renormalization of higher-derivative quantum gravity," Physical Review D, vol. 16, no. 4, pp. 953-969, 1977.

[5] N. D. Birrell and P. C. W. Davies, Quantum Fields in Curved Spacetime, Cambridge University Press, Cambridge, UK, 1982.

[6] I. L. Buchbinder, S. D. Odinstov, and I. L. Smapiro, Effective Actions in Quantum Gravity, IOP Publishing, Bristol, UK, 1992.

[7] A. de Felice and S. Tsujikawa, " $\mathrm{f}(\mathrm{R})$ theories," Living Reviews in Relativity, vol. 13, no. 3, pp. 1-161, 2010.

[8] L. P. Eisenhart, Riemannian Geometry, Princeton University Press, Princeton, NJ, USA, 1949.

[9] D. Grumiller, W. Kummer, and D. V. Vassilevich, Physics Reports, vol. 369, no. 4, Article ID 104012, pp. 327-430, 2002.

[10] C. G. Callan, S. B. Giddings, J. A. Harvey, and A. Strominger, "Evanescent black holes," Physical Review D, vol. 45, no. 4, pp. R1005-R1009, 1992.

[11] G. Mandal, A. M. Sengupta, and S. R. Wadia, "Classical solutions of two-dimensional string theory," Modern Physics Letters A, vol. 6, pp. 1685-1692, 1991.

[12] E. Witten, "String theory and black holes," Physical Review D, vol. 44, no. 2, pp. 314-324, 1991.

[13] M. A. Ahmed, "Classical and quantum cosmology in a model of two-dimensional dilaton gravity," Physical Review D, vol. 61, no. 10, Article ID 104012, 8 pages, 2000.

[14] M. A. Ahmed, "Cosmology in two-dimensional dilaton gravity theories," Nuovo Cimento della Societa Italiana di Fisica B, vol. 121, no. 7, pp. 661-673, 2006.

[15] S. Weinberg, Gravitation and Cosmology: Principles and Applications of the General Theory of Relativity, John Wiley \& Sons, New York, NY, USA, 1972.

[16] K. C. K. Chan and R. B. Mann, "Cosmological models in two spacetime dimensions," Classical and Quantum Gravity, vol. 10, no. 5, pp. 913-930, 1993.

[17] A. Vilenkin, "Classical and quantum cosmology of the Starobinsky inflationary model," Physical Review D, vol. 32, no. 10, pp. 2511-2521, 1985.

[18] M. P. Hobson, G. Efstathiou, and A. N. Lasenby, General Relativity: An Introduction for Physicists, Cambridge University Press, Cambridge, UK, 2006.

[19] A. R. Liddle and D. H. Lyth, Cosmological Inflation \& Large Scale Structure, Cambridge University Press, Cambridge, UK, 2000.

[20] S. W. Hawking and J. C. Luttrell, "Higher derivatives in quantum cosmology. (I). The isotropic case," Nuclear Physics, Section B, vol. 247, no. 1, pp. 250-260, 1984.

[21] K. W. Ford, D. L. Hill, M. Wakano, and J. A. Wheeler, "Quantum effects near a barrier maximum," Annals of Physics, vol. 7, no. 3, pp. 239-258, 1959.

[22] W. A. Friedman and C. J. Goebel, "Barrier-top resonances and heavy ion reactions," Annals of Physics, vol. 104, no. 1, pp. 145183, 1977.

[23] G. Barton, "Quantum mechanics of the inverted oscillator potential," Annals of Physics, vol. 166, no. 2, pp. 322-363, 1986.

[24] N. L. Balazs and A. Voros, "Wigner's function and tunneling," Annals of Physics, vol. 199, no. 1, pp. 123-140, 1990.

[25] D. Chruściński, "Quantum mechanics of damped systems. II. Damping and parabolic potential barrier," Journal of Mathematical Physics, vol. 45, no. 3, pp. 841-854, 2004. 
[26] M. Abramowitz and I. Stegun, Handbook of Mathematical Functions, Dover, New York, NY, USA, 1972.

[27] Z. X. Wong and D. R. Guo, Special Functions, World Scientific, Singapore, 1989.

[28] A. A. Starobinsky, "A new type of isotropic cosmological models without singularity," Physics Letters B, vol. 91, no. 1, pp. 99-102, 1980. 

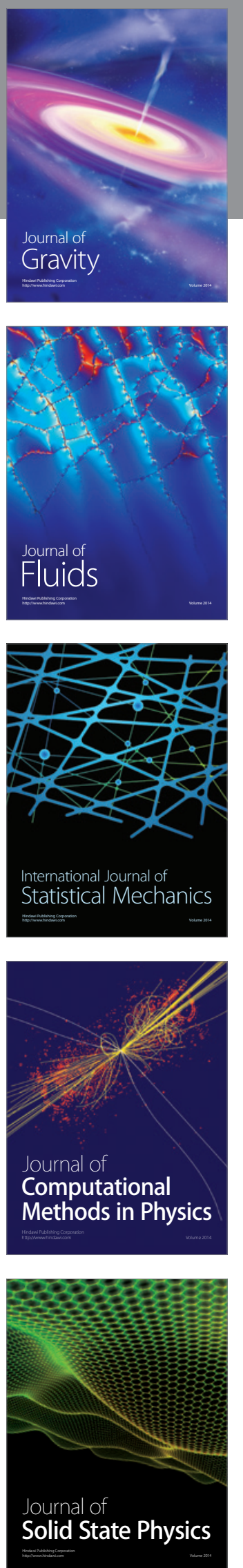

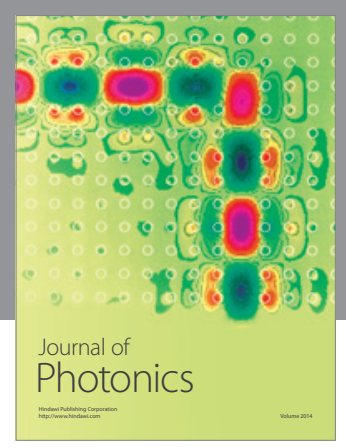

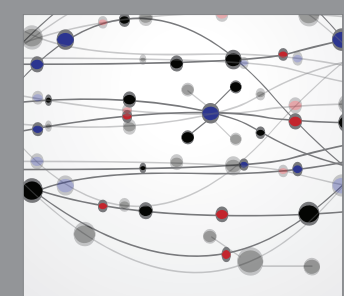

The Scientific World Journal
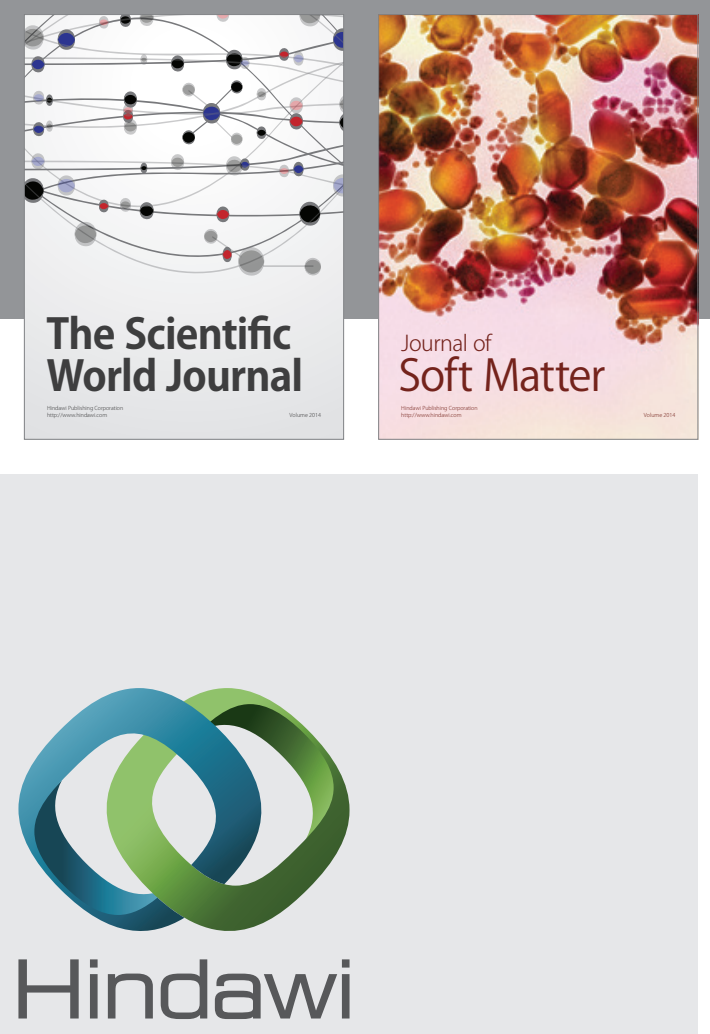

Submit your manuscripts at

http://www.hindawi.com
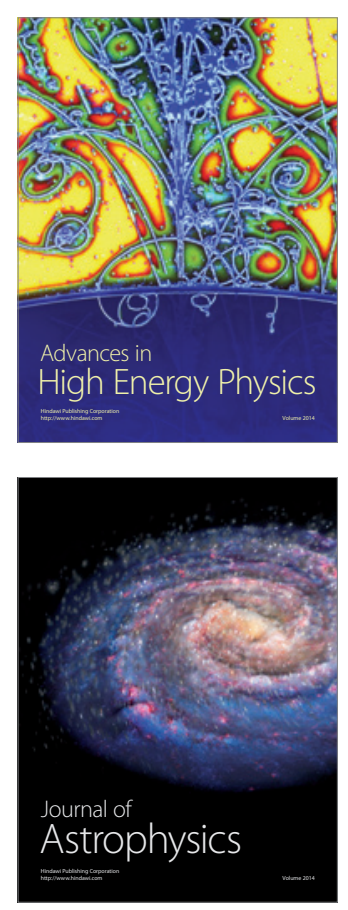
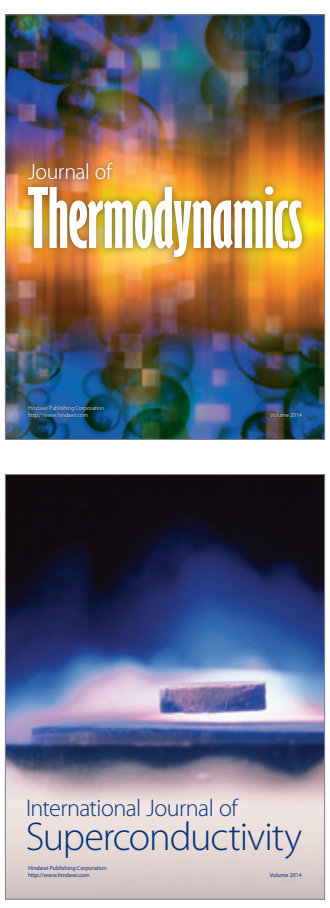
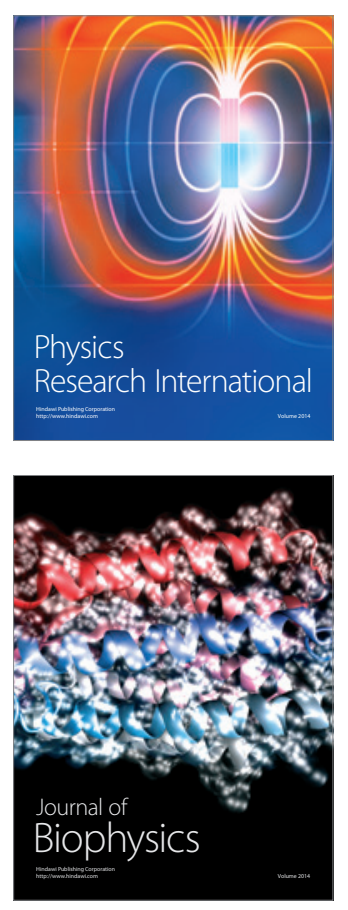
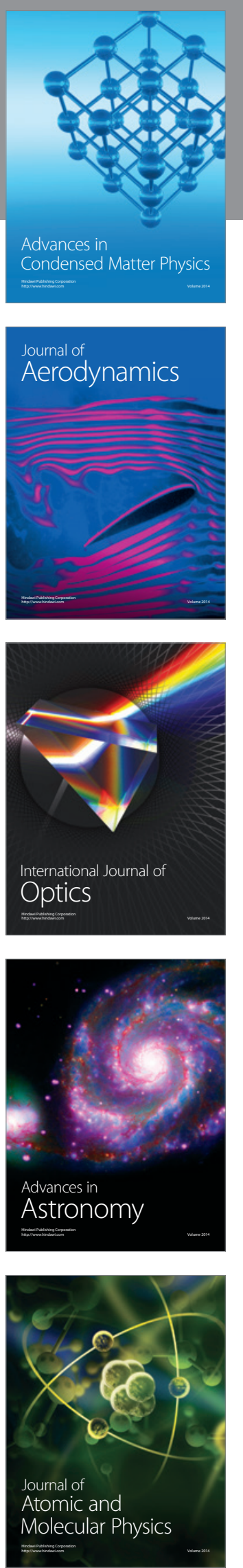